\title{
Correction to: Percutaneous Deep Venous Arterialization for Severe Critical Limb Ischemia in Patients With No Option of Revascularization: Early Experience From Two European Centers
}

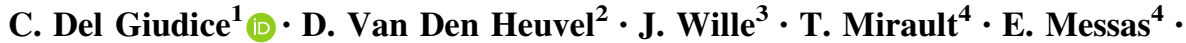 \\ R. Ferraresi ${ }^{5} \cdot$ S. Kum ${ }^{6}$ - M. Sapoval ${ }^{1}$
}

Published online: 30 July 2018

(C) Springer Science+Business Media, LLC, part of Springer Nature and the Cardiovascular and Interventional Radiological Society of Europe (CIRSE) 2018

\section{Correction to:}

\section{Cardiovasc Intervent Radiol}

https://doi.org/10.1007/s00270-018-2020-2

The fifth author's name was incorrectly published as "M. Messas". The correct name is "E. Messas". The original article has been corrected.

The original article can be found online at https://doi.org/10.1007/ s00270-018-2020-2.

C. Del Giudice

costantino.delgiudice@gmail.com

1 Vascular and Oncological Interventional Radiology, Hôpital Européen Georges-Pompidou, Université Paris Descartes Paris Cité Sorbonne, 20 Rue Leblanc, 75015 Paris, France

2 Department of Interventional Radiology, St Antonius Hospital, Nieuwegein, The Netherlands

3 Department of Vascular Surgery, St Antonius Hospital, Nieuwegein, The Netherlands

4 Department of Vascular Medicine, Hôpital Européen Georges Pompidou, Université Paris Descartes Paris Cité Sorbonne, 20 Rue Leblanc, 75015 Paris, France

5 Peripheral Interventional Unit, Humanitas Gavazzeni, Bergamo, Italy

6 Vascular Service, Department of Surgery, Changi General Hospital, Changi, Singapore 